Editorial

\title{
Acknowledgment to Reviewers of Diseases in 2021
}

\section{Diseases Editorial Office}

Citation: Diseases Editorial Office.

Acknowledgment to Reviewers of Diseases in 2021. Diseases 2022, 10, 8 . https://doi.org/10.3390/

diseases 10010008

Published: 27 January 2022

Publisher's Note: MDPI stays neutral with regard to jurisdictional claims in published maps and institutional affiliations.

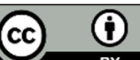

Copyright: ( 2022 by the authors. Licensee MDPI, Basel, Switzerland. This article is an open access article distributed under the terms and conditions of the Creative Commons Attribution (CC BY) license (https://creativecommons.org/licenses/by/4.0/).

MDPI AG, St. Alban-Anlage 66, 4052 Basel, Switzerland

Rigorous peer-reviews are the basis of high-quality academic publishing. Thanks to the great efforts of our reviewers, Diseases was able to maintain its standards for the high quality of its published papers. Thanks to the contribution of our reviewers, in 2021, the median time to first decision was 21 days, and the median time to publication was 43.5 days. The editors would like to extend their gratitude and recognition to the following reviewers for their precious time and dedication, regardless of whether the papers they reviewed were finally published:

Abdeen, Ahmed

Agüero-Chapin, Guillermin

Ahmed, Heba

Alcántara-Ortigoza, Miguel Angel

Alfie, José

Amero, Carlos

Andreoni, Francesca

Arenas, Roberto

Armesto Alonso, Susana

Arrivillaga, Jazzmin

Atanasova, Kalina

Avin, Vijay

Baldi, Ileana

Ballestri, Stefano

Balogh, Enikő

Bao, Xiucong

Barnea, Itay

Behringer, Erik

Bel'skaya, Lyudmila

Belosludtseva, Natalia V.

Benítez Sillero, Juan De Dios

Bennasar, Mar

Bojarska-Junak, Agnieszka A.

Brady, Gareth

Brasic, James

Brooke, Joanne

Brown, Gregory

Brown, Ronald B.

Buckingham, Lela

Caccamo, Daniela

Cai, Qiangjun

Căinap, Călin

Canales, Jimena

\author{
Carlisi, Daniela \\ Carta, Angela \\ Chela, Harleen \\ Cho, Joo-Youn \\ Coimbra, Ibsen B. \\ Corea, Francesco \\ Costa, Solange \\ Costa-Bauzá, Antònia \\ Csaderova, Lucia \\ Currò, Monica \\ David, González-Barrio \\ Davis, David A. \\ Di Marco, Roberto \\ Duc, Nguyen Minh \\ El-Sheikh Ali, Hossam \\ Espinosa, Emma \\ Esteban, Luis Mariano \\ Fatone, Gerardo \\ Fenoglio, Roberta \\ Ferreira, Nelson \\ Fiering, Steve N. \\ Fife-Schaw, Chris \\ Franchini, Massimo \\ Franco, Roberto $S$. \\ Frías-De-León, María Guadalupe \\ Fulmer, Brant R. \\ Gamarra, Lionel Fernel \\ Garinis, Angela C. \\ Gatzoulis, Konstantinos A. \\ George, Anderson \\ Ghosh, Arjun Kumar \\ Godman, Brian \\ Gómez-Barrena, Enrique
}


Gozes, Illana

Granata, Antonio

Haque, Muhammad

Heijman, Jordi

Hernández-Vara, Jorge

Hildreth, Blake

Hong, Guoju

Horng, Chi-Ting

Horowitz, John D.

Horowitz, Robert A.

Houser, Madelyn C.

Huang, Fang

Jardini, Maria Aparecida Neves

Jiang, Jhih-Hang

Johanning, Eckardt

Jovanov-Milošević, Nataša

Jung, Ji Hoon

Kala, Rishabh

Kang, Jeonghyun

Kanno, Takeshi

Kano, Satoshi

Kidd, Lisa A.

Kleczyński, Paweł

Kobeissy, Firas

Koyama, Sachiko

Krams, Indrikis

Krela-Kaźmierczak, Iwona

Kucukseymen, Selcuk

Kuliczkowski, Kazimierz

Lasithiotakis, Konstantinos

Lee, Chii-Ming

Leng, Gareth

Li, Mingqiang

Li, Sunan

Lichtenauer, Michael

Lima, Marcelo

Lisotti, Andrea

Liu, Chung-Jung

Loretz, Brigitta

Lucke-Wold, Brandon

Luigi, Lavorgna

Macaluso, Giusi

Magrassi, Lorenzo

Mallmann, Michael Rudolf

Mani, Alireza

Mathew, Rajamma

Mathieu, David

Mauri, Davide

Meggyes, Mátyás

Meng, Yan

Monroy, Fernando
Moon, Jee-Young

Moreira, Mário W. L.

Morford, Lorri

Naldi, Luigi

Oftadeh, Shahin

Ogino, Shuji

Olschewski, Horst

Owczarczyk-Saczonek, Agnieszka

Panzuto, Francesco

Paradkar, Prasad

Parkar, Shanthi G.

Peh, Hong Yong

Peitsidis, Panagiotis

Pellegrinelli, Laura

Peres, Nalu Teixeira Aguiar

Peserico, Alessia

Peters, John C.

Picó-Monllor, José Antonio

Pieper, Barbara

Pintos-Morell, Guillem

Pogreba-Brown, Kristen

Polovic, Natalija

Pontes, Bruno

Pop, Dana

Prats, Anne-Catherine

Pripp, Are Hugo

Pyza, Elżbieta

Rangel, Érika B.

Razvan-Cosmin, Petca

Reyes-Ortega, Felisa

Richardson, Sandra R.

Rocchiccioli, Silvia

Romani, Patrizia

Rossi, Alessandro

Roussakow, Sergey V.

Routledge, Michael

Ruan, Ke

Russo, Antonio

Salucci, Sara

Sanchez, Maria T.

Sarid, Orly

Sato, Eiichi

Serre, Karine

Shen, Ming-Yi

Shoshkes-Carmel, Michal

Siqueira, Hugo Valadares

Sivula, Mirka

Smaga, Irena

Song, Mi-Yeon

Soreq, Hermona

Spennato, Pietro 
Stabile, Guglielmo

Stellas, Dimitris

Stricker, Hans

Stridh, Pernilla

Subbiah, Murugan

Szeliga, Monika

Tabachnik Schor, Nina F.

Takahashi, Tetsufumi

Takala, Jukka

Taradaj, Jakub

Téllez-Valencia, Alfredo

Tesarik, Jan

Toietta, Gabriele

Tonhajzerova, Ingrid

Uzunoglu, Faik G.

Vafiadaki, Elizabeth
Vandenbroeck, Koen

Veranic, Peter

Viana, Joao Henrique Moreira

Wang, Hsiuying

Wang, Kai

Wennmann, Jörg

Wiedmann, Felix

Wróbel, Agnieszka

Wu, Meng-Yu

$\mathrm{Xu}$, Yan-Ming

Yatomi, Masakiyo

Yeligar, Samantha M.

Yen, Cheng-Fang

Zaman, Shafquat

Zarobkiewicz, Michał

Zingg, Jean-Marc 